\title{
Evaluación del Efecto del Tratamiento con Acido Lipoico Administrado a la Rata Wistar Intoxicada con el Fruto de Karwinskia humboldtiana
}

\author{
Evaluation of the Effect of Treatment with Lipoic Acid Administered to \\ the Wistar Rat Intoxicated with the Fruit of Karwinskia humboldtiana \\ *Jaime García-Juárez; **:Martha Elizabeth Salazar-Leal; ***:Santos Guzmán-López; \\ "Víktor Javier Romero-Díaz; ${ }^{* * * * *}$ Rosalba Ramírez-Durón \& *Julio Sepúlveda-Saavedra
}

GARCÍA-JUÁREZ, J.; SALAZAR-LEAL, M. E.; GUZMÁN-LÓPEZ, S.; ROMERO-DÍAZ, V. J.; RAMÍREZ-DURÓN, R. \& SEPÚLVEDA-SAAVEDRA, J. Evaluación del efecto del tratamiento con ácido lipoico administrado a la rata wistar intoxicada con el fruto de Karwinskia humboldtiana. Int. J. Morphol., 30(2):572-578, 2012.

RESUMEN: La ingesta accidental de fruto de Karwinskia humboldtiana ocasiona una parálisis flácida, simétrica, progresiva y ascendente, similar al síndrome de Guillain-Barré. Evoluciona en el transcurso de 3 a 12 meses hasta su recuperación total, pero los casos graves terminan en la muerte por insuficiencia respiratoria. No existe un tratamiento específico. La lesión histopatológica descrita en nervio periférico de pacientes, y animales de experimentación corresponde a una desmielinización segmentaria acompañada de degeneración Walleriana. Una de las toxinas extraídas a partir de la semilla, la T-514, ocasiona un incremento de radicales libres in vitro. Los radicales libres se han relacionado con la desmielinización que se presenta en otros tipos de neuropatías como en la diabética. Ya que la lesión ultraestructural que se presenta en los modelos animales de diabetes es similar a la que se observa en la intoxicación experimental con fruto de K. humboldtiana, se decidió administrar un potente agente antioxidante, el ácido $\alpha$-lipoico en un modelo de intoxicación crónica por fruto de K. humboldtiana. Sin embargo, no se observó mejoría sobre las manifestaciones clínicas evaluadas en los animales o sobre las lesiones histopatológicas presentes en el nervio periférico. Estos resultados sugieren que los radicales libres no son el mecanismo principal de lesión sobre el nervio periférico en la polineuropatía causada por K. humboldtiana.

PALABRAS CLAVE: Karwinskia humboldtiana; Ácido lipoico; Polineuropatía; Desmielinización; Parálisis.

\section{INTRODUCCIÓN}

Karwinskia humboldtiana $(K h)$, comúnmente conocida como "capulincillo", "tullidora" o "coyotillo", es un arbusto venenoso que crece a lo largo de toda la República Mexicana y al sur de los Estados Unidos de América (Fernández-Nava, 1992). La ingesta accidental de fruto de $K h$ por animales y por humanos (Padrón-Puyou, 1951), ocasiona una parálisis flácida, simétrica, progresiva y ascendente, similar al síndrome de Guillain-Barré (García-Ramos \& Cacho-Díaz, 2005), con el cual se confunde. El cuadro neurológico, evoluciona generalmente en el transcurso de 3 a 12 meses hasta su recuperación total, dependiendo de la cantidad ingerida (Padrón-Puyou, 1951; Padrón \& Velázquez, 1956), pero los casos graves terminan en la muerte por insuficiencia respiratoria y no existe un tratamiento específico (Puértolas et al., 1984). La lesión histopatológica descrita en nervio periférico de pacientes y de animales de experimentación se caracteriza por una desmielinización segmentaria acompañada por degeneración Walleriana del axón (Escobar \& Nieto, 1965; Del Pozo, 1965; CalderónGonzález \& Rizzi-Hernández, 1967). También se ha descrito un incremento en el número de células cebadas presentes en el endoneurio (Becerra, et al., 2005). Recientemente se desarrolló un modelo de intoxicación crónica con dosis fraccionadas de fruto molido, en el que se logró reproducir el cuadro neurológico y las lesiones histopatológicas que se han reportado en los seres humanos. En este modelo se describe la intoxicación antes y durante el desarrollo del cuadro neurológico así como la evolución hasta su recuperación (Salazar-Leal et al., 2006). Además, a partir de la semilla de este fruto, se han aislado varios compuestos

\footnotetext{
* Departamento. de Histología, Facultad de Medicina, Universidad Autónoma de Nuevo León, Monterrey, N. L., México.

** Departamento. de Farmacología y Toxicología, Facultad de Medicina, Universidad Autónoma de Nuevo León, Monterrey, N. L., México.

*** Departamento de Anatomía, Facultad de Medicina, Universidad Autónoma de Nuevo León, Monterrey, N. L., México.

*** Departamento de Química Analítica, Facultad de Medicina, Universidad Autónoma de Nuevo León, Monterrey, N. L., México.
} 
químicos antracenónicos tóxicos, clasificados de acuerdo a su peso molecular como: T-496, T-514, T-516 y T-544 (Dreyer et al., 1975), entre otras. Estudios in vitro con la T514 renombrada peroxisomicina A1 (Sepúlveda-Saavedra et al., 1992), mostraron un incremento de especies reactivas de oxígeno cuando se agrega al medio de cultivo de una monocapa (Garza-Ocañas et al., 2003). El incremento de radicales libres se ha relacionado con la desmielinización que se presenta en otros tipos de neuropatías, como la diabética (Evans et al., 2002). Así mismo, la lesión ultraestructural que se presenta en los modelos animales de diabetes inducida por estreptozotocina (Fahim et al., 2000) es similar a la que se observa en la intoxicación experimental por fruto de $K h$ (Salazar-Leal et al.). Existen reportes de un efecto favorable sobre la neuropatía diabética cuando se administran a estos pacientes agentes antioxidantes como el ácido $\alpha$-lipoico, el cual, además de depurar a los radicales libres, tiene la capacidad de restablecer a los sistemas antioxidantes endógenos (Biewenga et al., 1997; Ziegler et al., 1999). En los modelos experimentales de diabetes, el ácido $\alpha$-lipoico mejora la conducción nerviosa y previene el desarrollo de la neuropatía (Pitel et al., 2007).

En el presente trabajo se evaluó el efecto del ácido $\alpha$-lipoico sobre las manifestaciones clínicas y las lesiones histológicas que se presentan en nervio periférico en un modelo de intoxicación crónica por fruto de $K h$ en la rata (Salazar-Leal et al.).

\section{MATERIAL Y MÉTODO}

Colecta y preparación del fruto molido. El fruto maduro de $K h$ se recolectó en la localidad de Hidalgo, N.L., México, durante los meses de julio y agosto; el fruto fue secado, molido, tamizado y preparado en suspensión acuosa para su administración mediante una sonda orogástrica según el modelo desarrollado por Salazar et al.

Grupos de trabajo y tratamientos. Para este estudio se utilizaron 40 ratas Wistar de ambos sexos y de $250 \pm 25 \mathrm{~g}$, se mantuvieron bajo condiciones estándar de laboratorio y con ciclos de luz-oscuridad controlados de 12 horas c/u. Los animales se distribuyeron en grupos de 5 ratas c/u, los cuales se trataron en dos modalidades según la Tabla I. Para la primer modalidad se administró una dosis de $30 \mathrm{mg} / \mathrm{Kg}$ /día de ácido $\alpha$-lipoico (Sigma-Aldrich Co. St. Louis, MO, T5625 lote $069 \mathrm{~K} 0308$ ) disuelto en aceite de maíz, por vía oral (vo) durante 15 días previos a la administración del fruto de $K h$. Para la modalidad de tratamiento posterior a la administración de fruto molido de $K h$ se administraron $30 \mathrm{mg} / \mathrm{Kg}$ /día de ácido $\alpha$-lipoico (Thioctacid $®$ 600T Bayer lote X102), en solución salina por vía intraperitoneal (ip) a partir del día 14 posterior a la primer dosis de fruto y, hasta el día 58, cuando se presenta la parálisis en los animales del grupo control que no recibieron ácido $\alpha$-lipoico. La dosis total de fruto molido administrado fue de $3,5 \mathrm{~g} / \mathrm{Kg}$ de peso, fraccionada en 5 tomas, los días $0,3,7,10$ y 14; la primera dosis fue de $1,5 \mathrm{~g} / \mathrm{Kg}$ de peso y las cuatro siguientes de $0,5 \mathrm{~g} / \mathrm{Kg}$ de peso. Para cada modalidad de tratamiento se contó con grupos control (Tabla I).

Colecta de las muestras. Todos los animales fueron sacrificados con pentobarbital (Anestesal® Pfizer SAGARPA Q0001-065) a una dosis de $40 \mathrm{mg} / \mathrm{Kg}$ de peso por vía ip, al día 58 posterior a la administración de la primera dosis del fruto. Los animales fueron perfundidos con $125 \mathrm{ml}$ de una solución de glutaraldehído al $2.5 \%$ y paraformaldehído al 4 $\%$ en buffer de fosfatos a $\mathrm{pH}$ 7,2 por vía intracardíaca durante $20 \mathrm{~min}$. Posteriormente se obtuvieron los nervios ciáticos y safenos, que fueron fijados en glutaraldehído al $2,5 \%$ en buffer de cacodilatos a $\mathrm{pH} 7,2$.

Procesamiento histológico de las muestras. Todas las muestras fueron procesadas mediante la técnica histológica de rutina e incluidas en bloques de parafina. Se realizaron secciones histológicas de $5 \mu \mathrm{m}$ teñidas con hematoxilina y eosina, de $7 \mu \mathrm{m}$ teñidas mediante la técnica de Klüver-Barrera y, de $10 \mu \mathrm{m}$ tratadas mediante el método de Marsland, Glees y Erickson.

\section{RESULTADOS}

Las ratas de los grupos A-AAL, A-AAL, AM-A y ASS mostraron una curva de crecimiento normal, mientras que los grupos AAL- $K h$, $K h$-AAL, AM- $K h$ y $K h$-SS mostraron disminución de peso más notable a partir de la cuarta semana posterior a la administración del fruto de $K h$. Los animales del grupo AAL- $K h$ no mostraron diferencia alguna con respecto al grupo AM- $K h$, así como tampoco se observó diferencia entre los grupos $K h$-AAL y $K h$-SS $(\mathrm{p}=0,1675)$. Las manifestaciones clínicas que presentaron los animales de los grupos que recibieron fruto de $K h$ (AAL$K h, K h$-AAL, AM- $K h$ y $K h$-SS), están registradas en la Tabla II. Cabe señalar, que los tratamientos con ácido $\alpha$-lipoico, ya sea administrado previo o posterior a la administración de fruto de $K h$, no tuvieron ningún efecto favorable sobre alguna de las manifestaciones registradas. Los animales del grupo $K h$-AAL mostraron agravamiento de algunas de las manifestaciones, tales como la disminución de la actividad espontánea, debilidad, secreción de porfirina periocular y ataque al estado general (Tabla II). 
GARCÍA-JUÁREZ, J.; SALAZAR-LEAL, M. E.; GUZMÁN-LÓPEZ, S.; ROMERO-DÍAZ, V. J.; RAMÍREZ-DURÓN, R. \& SEPÚLVEDA-SAAVEDRA, J. Evaluación del efecto del tratamiento con ácido lipoico administrado a la rata wistar intoxicada con el fruto de Karwinskia humboldtiana. Int. J. Morphol., 30(2):572-578, 2012.

Tabla I. Grupos de trabajo y tratamientos.

\begin{tabular}{ll}
\hline Grupos & \multicolumn{1}{c}{ Tratamientos } \\
\hline AAL-Kh & Acido $\alpha$-lipoico en aceite de maíz vo durante 15 días previo a Kh \\
$\boldsymbol{K} \boldsymbol{h}$-AAL & Acido $\alpha$-lipoico en solución salina ip posterior a Kh desde el día 14 al 58 \\
AM- $\boldsymbol{h} \boldsymbol{h}$ & Aceite de maíz por vo durante 15 días previo a Kh \\
$\boldsymbol{K} \boldsymbol{h}$-SS & Solución salina ip posterior a Kh desde el día 14 al 58 \\
AAL-AM & Acido $\alpha$-lipoico en aceite de maíz por vo durante 15 días \\
A-AAL & Acido $\alpha$-lipoico en solución salina ip desde el día 14 al 58 \\
AM-A & Aceite de maíz vo durante 15 días \\
A-SS & Solución salina ip desde el día 14 al 58 \\
\hline
\end{tabular}

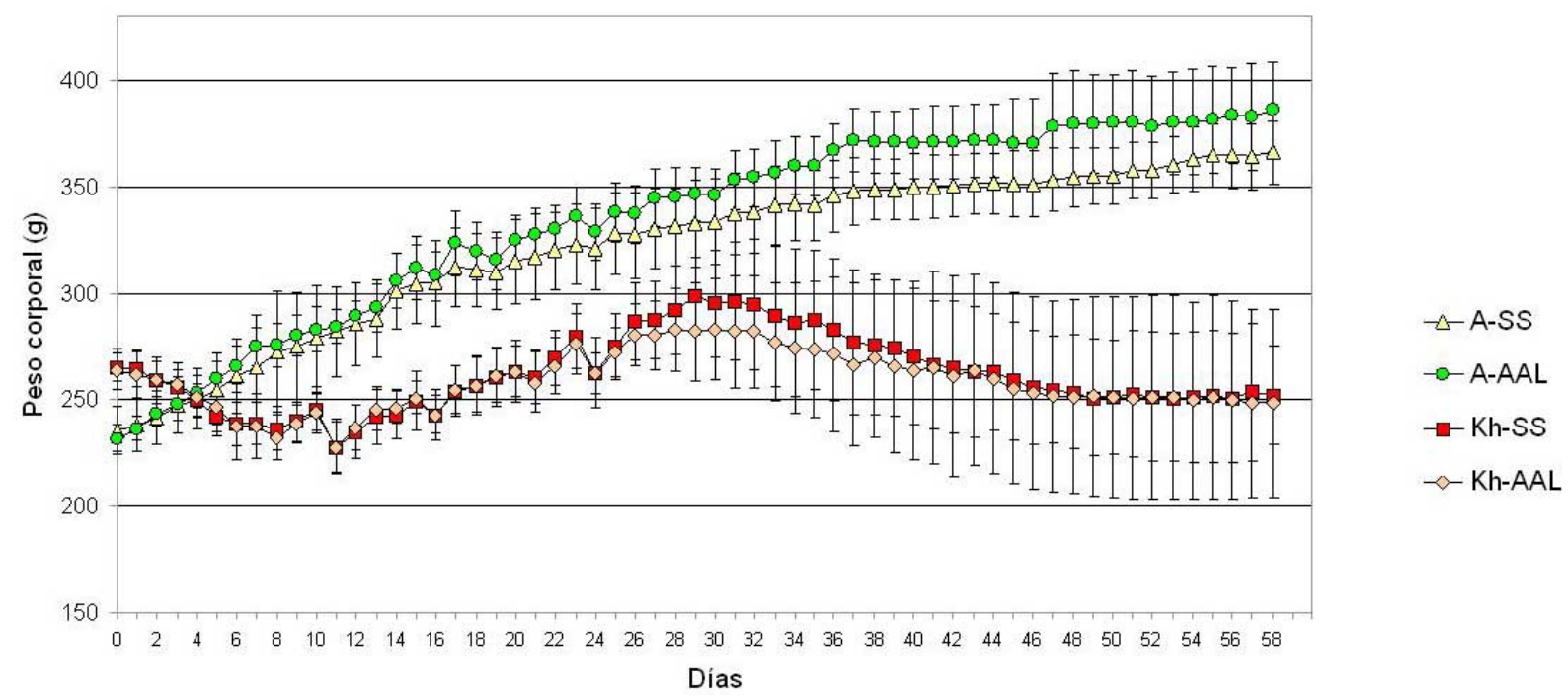

Fig. 1. Curvas de peso de las ratas que recibieron ácido $\alpha$-lipoico por vía ip desde el día 14 al 58 posteriores a la intoxicación con fruto de $K h(K h-\mathrm{AAL})$ y sus respectivos grupos control, p=0,1675.
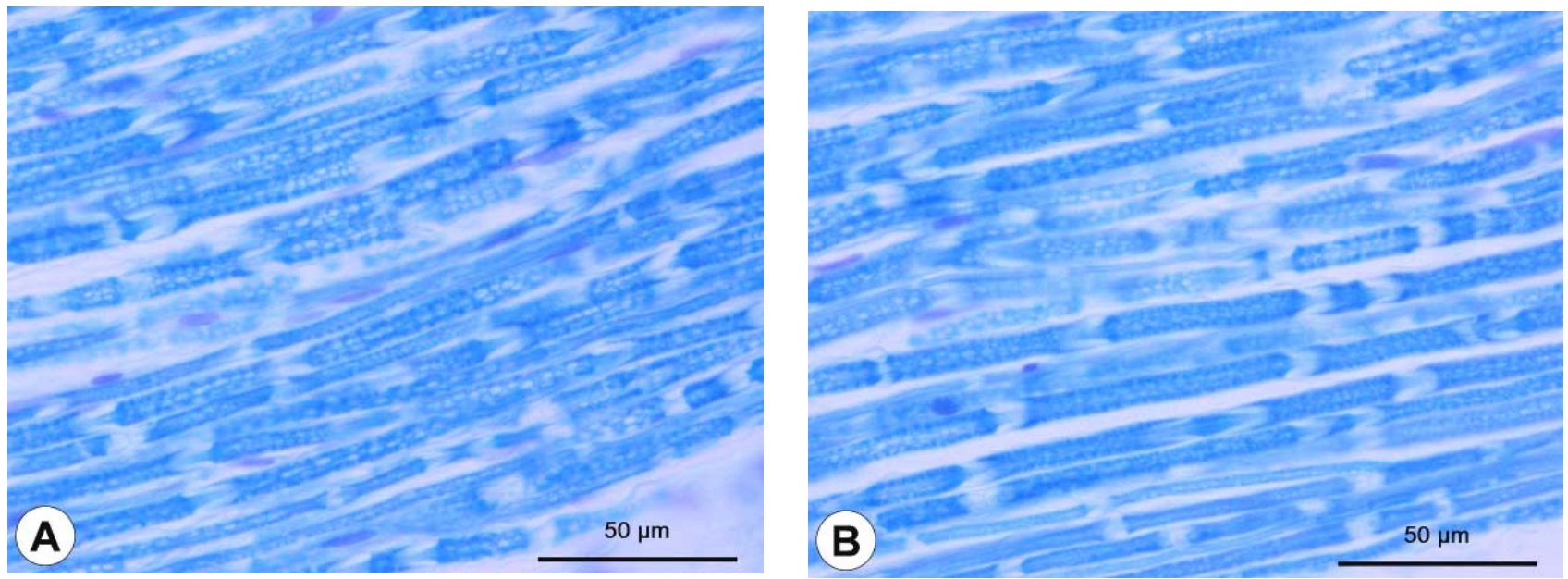
GARCÍA-JUÁREZ, J.; SALAZAR-LEAL, M. E.; GUZMÁN-LÓPEZ, S.; ROMERO-DÍAZ, V. J.; RAMÍREZ-DURÓN, R. \& SEPÚLVEDA-SAAVEDRA, J. Evaluación del efecto del tratamiento con ácido lipoico administrado a la rata wistar intoxicada con el fruto de Karwinskia humboldtiana. Int. J. Morphol., 30(2):572-578, 2012.
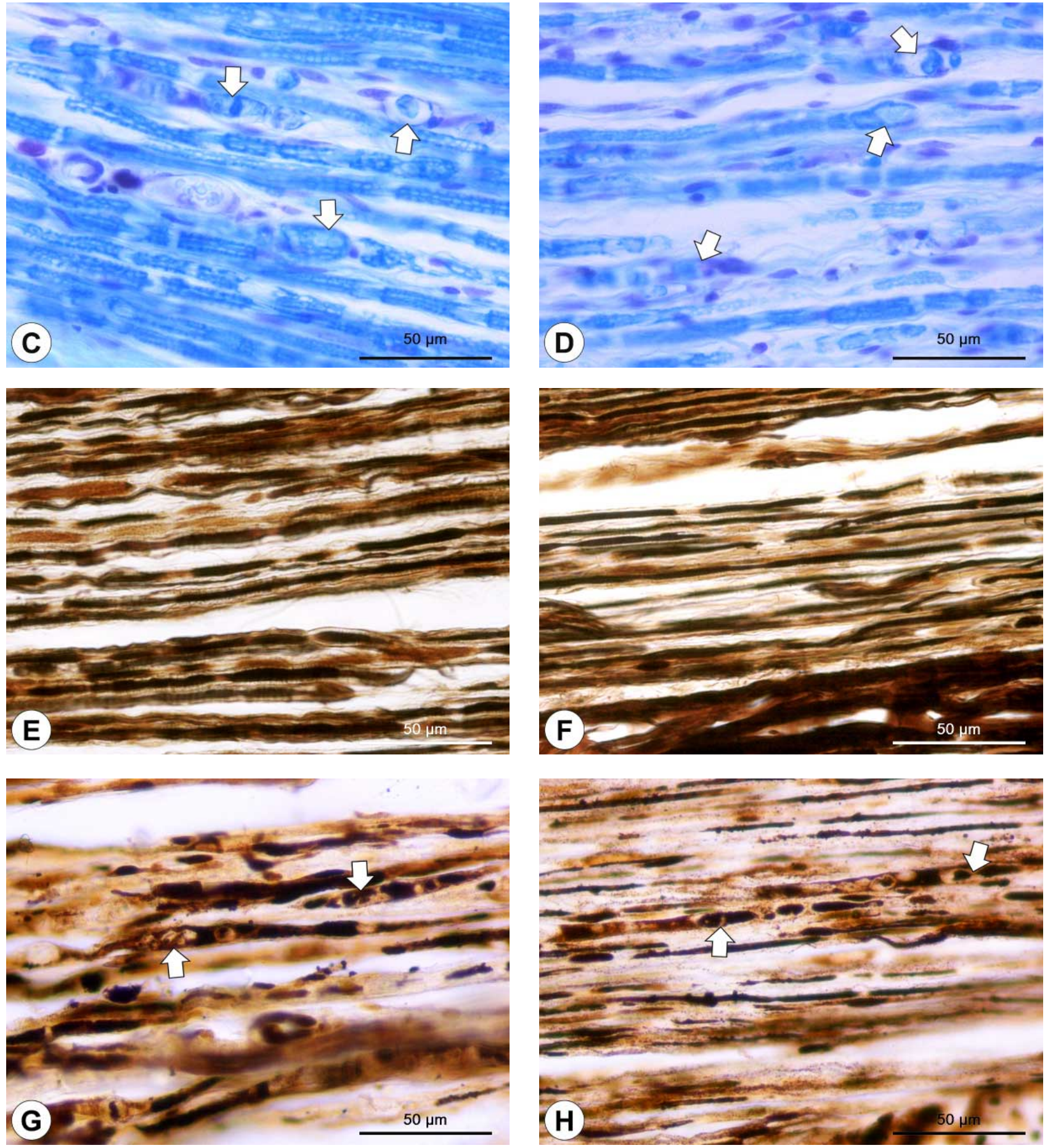

Fig. 2. A. Nervio periférico de una rata del grupo A-SS. Se observan las vainas de mielina en color azul y de aspecto normal. B. Nervio periférico, rata del grupo A-AAL. Se aprecia un aspecto normal. C. Corte de nervio periférico de una rata del grupo Kh-SS. Se observa pérdida y fragmentación de la mielina en segmentos de las fibras nerviosas, presencia de macrófagos por dentro de las mismas (flechas). D. Nervio periférico de una rata del grupo Kh-AAL. Se aprecian las mismas alteraciones (flechas). Cortes histológicos de $7 \mu \mathrm{m}$ de grosor, teñidos mediante la técnica de Klüver-Barrera, 40 x. E. Nervio periférico de una rata del grupo control A-SS. Se observan el trayecto y grosor regulares de los axones. F. Nervio periférico de una rata del grupo control A-AAL. Muestra axones de aspecto normal. G. Nervio del grupo $K h$-SS. Obsérvese la presencia de segmentos que presentan degeneración Walleriana (flechas). H. Nervio del grupo $K h$-AAL. Se observa degeneración Walleriana (flechas). Cortes histológicos de $10 \mu \mathrm{m}$ de grosor, impregnados con plata. Técnica de Marsland, Glees y Erickson; 40 x. 
Tabla II. Manifestaciones clínicas registradas en las ratas de cada grupo. Manifestaciones clínicas: A) Piloerección, B) dificultad respiratoria, C) disminución de la actividad espontánea, D) debilidad, E) alteración de la marcha, F) atrofia muscular, G) mano en garra, H) deformidad espinal, I) secreción de porfirina periocular, J) ataque al estado general. Escala: ausente ( - ), presente $(+)$, moderada $(++)$ y grave $(+++)$.

\begin{tabular}{lcccccccccc}
\hline \multirow{2}{*}{ Grupos } & \multicolumn{1}{c}{ Manifestaciones clínicas } \\
\cline { 2 - 9 } & A & B & C & D & E & F & G & H & I & J \\
\hline AAL-Kh & + & + & + & + & + & + & + & + & + & + \\
Kh-AAL & + & + & +++ & +++ & + & + & + & + & +++ & +++ \\
AM-Kh & + & + & + & + & + & + & + & + & + & + \\
Kh-SS & + & + & + & + & + & + & + & + & + & + \\
AAL-AM & - & - & - & - & - & - & - & - & - & - \\
A-AAL & - & - & - & - & - & - & - & - & - & - \\
AM-A & - & - & - & - & - & - & - & - & - & - \\
A-SS & - & - & - & - & - & - & - & - & - & - \\
\hline
\end{tabular}

Evaluación histológica. Los nervios ciáticos y safenos de los grupos AAL-AM, A-AAL, AM-A y A-SS presentaron un trayecto y grosor regulares, el endoneurio también presentó un aspecto normal (Figs. 2A, 2B, 2E y 2F). En los grupos AAL- $K h, K h$-AAL, AM- $K h$ y $K h$-SS se observó desmielinización segmentaria y degeneración Walleriana del axón (Figs. 2C, 2D, 2G y 2H), además se observó la presencia de macrófagos y un aparente incremento de células cebadas adentro del endoneurio.

\section{DISCUSIÓN}

El modelo de intoxicación crónica que se empleó en este trabajo (Salazar-Leal et al.) resulta muy sencillo de manejar y además reproduce las manifestaciones neurológicas descritas en los pacientes intoxicados (PadrónPuyou). Esto resulta particularmente útil para estudiar el posible mecanismo etiopatogénico que ocasiona el cuadro clínico y las lesiones histopatológicas de los órganos estudiados, ya que actualmente no se conoce (Puértolas et al.). Aunado a esto, el reporte de que una de las toxinas presentes en el fruto de $K h$, la T-514, ocasiona un incremento de radicales libres en monocapas celulares in vitro (GarzaOcañas et al.) nos motivó a evaluar, en este modelo, el uso de un agente antioxidante probado con eficacia en otras polineuropatías desmielinizantes, como la que se presenta en la diabetes (Biewenga et al.; Ziegler et al.; Pitel et al.). Sin embargo, en este tipo de neuropatía tóxica no se obser- vó ningún efecto benéfico aparente en las ratas que fueron tratadas con ácido $\alpha$-lipoico. En lugar de esto, se observó que algunas manifestaciones clínicas se agravaron (Tabla II). Este fenómeno podría ser propiciado porque la $K h$ ocasiona insuficiencia renal (Jaramillo-Juárez et al., 1995), además de lesión e insuficiencia hepática (Bermúdez et al., 1986; Jaramillo et al., 2009). Estos órganos se encargan normalmente del metabolismo y eliminación de los fármacos, pero en este caso; al estar comprometidas sus funciones debido a la intoxicación, no ocurre una adecuada eliminación del ácido $\alpha$-lipoico, lo que podría agravar el cuadro. Las alteraciones histopatológicas observadas en los nervios afectados concuerdan con las reportadas previamente en biopsias de pacientes intoxicados accidentalmente y en animales de experimentación (Padrón-Puyou; Calderón-González \& RizziHernández; Charlton \& Pierce, 1970; Salazar-Leal et al.). Estos resultados sugieren que las lesiones ocasionadas al nervio periférico por esta intoxicación no son causadas directa, ni principalmente, por un incremento en la producción de radicales libres, por lo que es necesario valorar la participación de otros mediadores causantes de esta patología. En nuestro laboratorio, actualmente evaluamos el efecto del ácido $\alpha$-lipoico sobre la lesión histológica de otros órganos del sistema nervioso central como cerebro, cerebelo y médula espinal, ya que se han reportado lesiones a neuronas y tractos de la vía motora, causadas por la administración del fruto molido de $K h$ (Becerra-Verdín et al., 2009). Así como sobre las lesiones que presentan el riñón, pulmón e hígado, en este modelo de intoxicación crónica (Romero-Díaz et al., 2005). 
GARCÍA-JUÁREZ, J.; SALAZAR-LEAL, M. E.; GUZMÁN-LÓPEZ, S.; ROMERO-DÍAZ, V. J.; RAMÍREZ-DURÓN, R. \& SEPÚLVEDA-SAAVEDRA, J. Evaluación del efecto del tratamiento con ácido lipoico administrado a la rata wistar intoxicada con el fruto de Karwinskia humboldtiana. Int. J. Morphol., 30(2):572-578, 2012.

\section{AGRADECIMIENTOS}

Se agradece al PAICYT 2009 por el apoyo financiero otorgado para la presente investigación y, al CONACYT por la beca para obtener el grado de Doctor en Ciencias. A la Sra. Nora Frías, Luis Botello y Lorenzo Rada por la ayuda técnica.

GARCÍA-JUÁREZ, J.; SALAZAR-LEAL, M. E.; GUZMÁN-LÓPEZ, S.; ROMERO-DÍAZ, V. J.; RAMÍREZ-DURÓN, R. \& SEPÚLVEDA-SAAVEDRA, J. Evaluation of the effect of treatment with lipoic acid administered to the wistar rat intoxicated with the fruit of Karwinskia humboldtiana. Int. J. Morphol., 30(2):572-578, 2012.

SUMMARY: The accidental ingestion of Karwinskia humboldtiana causes a flaccid, symmetrical, progressive and ascending paralysis, similar to Guillain-Barre syndrome. It evolves over the course of 3 to 12 months until full recovery, but severe cases end in death due to respiratory failure. There is no specific treatment. The histopathological lesions described in peripheral nerve of patients and in experimental animals, corresponds to segmental demyelination accompanied by Wallerian degeneration. One of the toxins extracted from the seed, T-514, causes an increase of free radicals in vitro. Free radicals have been associated to demyelination that occurs in other types of neuropathy such as diabetic neuropathy. Since the ultrastructural damage that occurs in animal models of diabetes is similar to that observed in experimental poisoning with the fruit of $K$. humboldtiana, we decided to administer a powerful antioxidant, $\alpha$-lipoic acid, in a model of chronic poisoning due of $K$. humboldtiana. However, no improvement was observed on the clinical manifestations evaluated in animals or in the histopathological lesions in the peripheral nerve. These results suggest that free radicals are not the primary mechanism of injury on the peripheral nerve caused by $K$. humboldtiana.

KEY WORDS: Karwinskia humboldtiana; Lipoic acid; Polyneuropathy; Demyelination; Paralysis.

\section{REFERENCIAS BIBLIOGRÁFICAS}

Becerra, E.; Sepúlveda, J.; Piñeyro, A.; Salazar, M. E.; Romero, V. J.; Bermúdez, M. V. \& Flores, M. S. Histochemical and ultrastructural changes in the sciatic nerve of the rat with chronic poisoning with ground fruit of K. humboldtiana by oral route. Histol. Histopathol., Suppl. 1:S138, 2005.

Becerra-Verdín, E. M.; Bermúdez-Barba, M. V.; Salazar-Leal, M. E.; Ancer-Rodríguez, J.; Romero-Díaz, V.; Soto-Domínguez, A.; Ballesteros-Elizondo, R. G.; Saucedo-Cárdenas, O.; Piñeyro-López, A. \& Sepúlveda-Saavedra, J. Karwinskia humboldtiana (buckthorn) fruit causes central nervous system damage during chronic intoxication in the rat. Toxicon, 53(6):645-51, 2009.

Bermúdez, M. V.; González-Spencer, D.; Guerrero, M.; Waksman, N. \& Piñeyro, A. Experimental intoxication with fruit and purified toxins of buckthorn (Karwinskia humboldtiana). Toxicon, 24(11-12):1091-7, 1986.

Biewenga, G. P.; Haenen, G. R. \& Bast, A. The Pharmacology of the antioxidant lipoic acid. Gen. Pharmacol., 29(3):315-31, 1997.

Calderón-González R. \& Rizzi-Hernández, H. Buckthorn polyneuropathy. N. Engl. J. Med., 277(2):69-71, 1967.

Charlton, K. M. \& Pierce, K. R. A neuropathy in goats caused by experimental coyotiool (Karwinskia humboldtiana) poisoning. II. Lesions in the peripheral nervous system--teased fiber and acid phosphatase studies. Pathol. Vet., 7(5):385-407, 1970.
Del Pozo, E. C. Los efectos paralizantes de la "tullidora”. Estudios clínicos y experimentales. (Comentario al trabajo "Aspectos neuropatológicos de la intoxicación con Karwinskia humboldtiana. Estudio experimental."). Gac. Med. Mex., 95:179-82, 1965.

Dreyer, D. L.; Arai, I.; Bachman, C. D.; Anderson, W. R. Jr.; Smith, R. G. \& Daves, G. D. Jr. Toxins causing noninflammatory paralytic neuronopathy. Isolation and structure elucidation. $J$. Am. Chem. Soc., 97(17):4985-90, 1975.

Escobar A. \& Nieto D. Aspectos neuropatológicos de la intoxicación con Karwinskia humboldtiana. Estudio experimental. Gac. Med. Mex., 95(2):163-82, 1965.

Evans J. L.; Goldfine, I. D.; Maddux, B. A. \& Grodsky, G. M. Oxidative Stress and Stress-Activated Signaling Pathways: A Unifying Hypothesis of Type 2 Diabetes. Endocr. Rev., 23(5):599$622,2002$.

Fahim, M. A.; Hasan, M. Y. \& Alshuaib, W. B. Early morphological remodeling of neuromuscular junction in a murine model of diabetes. J. Appl. Physiol., 89(6):2235-40, 2000.

Fernández-Nava, R. Nombres comunes, usos y distribución geográfica del género Karwinskia (Rhamnaceae) en México. An. Inst. Biol. Ser. Bot., Univ. Nal. Autón. México, 63(1):1-23, 1992.

García-Ramos, G. S. \& Cacho-Díaz, B. Síndrome de Guillain-Barré (SGB). Diagnóstico diferencial. Rev. Mex. Neuroci., 6(5):448-54, 2005. 
GARCÍA-JUÁREZ, J.; SALAZAR-LEAL, M. E.; GUZMÁN-LÓPEZ, S.; ROMERO-DÍAZ, V. J.; RAMÍREZ-DURÓN, R. \& SEPÚLVEDA-SAAVEDRA, J. Evaluación del efecto del tratamiento con ácido lipoico administrado a la rata wistar intoxicada con el fruto de Karwinskia humboldtiana. Int. J. Morphol., 30(2):572-578, 2012.

Garza-Ocañas, L.; Zanatta-Calderón, M. T.; Acosta, D.; Torres-Alanís O. \& Piñeyro-López, A. Production of reactive oxygen species by toxin T-514 of genus Karwinskia in vitro. Toxicol. In Vitro, $17(1): 19-25,2003$.

Jaramillo-Juárez, F.; Ortíz, G.; Rodríguez-Vázquez, M. L.; FalcónFranco, M. A. \& Feria-Velazco, A. Renal failure during acute toxicity produced by tullidora ingestion (Karwinskia humboldtiana). Gen. Pharmac., 26(3):649-53, 1995.

Jaramillo, F.; Rodríguez, M. L.; Castillo, M. G.; Quezada, T.; Cerda, E.; Posadas, F. A. \& Taboada, C. Daño hepático y en la coagulación de la sangre producido por la administración de los frutos maduros de la planta tullidora (Karwinskia humboldtiana) en la rata. Rev. Mex. Cienc. Farm., 40(1):28-34, 2009.

Padrón, F. \& Velázquez, T. Patología experimental y clínica de la parálisis por Karwinskia humboldtiana. Rev. Mex. Ped., 25:22537, 1956.

Padrón-Puyou, F. Clinical and experimental studies on paralysis in children due to Karwinskia humboldtiana. Gac. Med. Mex., 81(23-4):299-311, 1951.

Pitel, S.; Raccah, D.; Gerbi, A.; Pieroni, G.; Vague, P. \& Coste, T. C. At low doses, a gamma-linolenic acid-lipoic acid conjugate is more effective than docosahexaenoic acid-enriched phospholipids in preventing neuropathy in diabetic rats. J. Nutr., 137(2):368-72, 2007.

Puértolas, M.; Nava, O.; Medina, H.; López, F. \& Oyervides, J. Polineuritis por Karwinskia humboldtiana. Rev. Med. IMSS, 22:22-7, 1984.

Romero-Díaz, V. J.; Salazar-Leal, M. E.; Flores-Diego, M. A.; Sepúlveda-Saavedra, J. \& Bermúdez-de Rocha, M. V. Morphologic study of lung lesion in a polyneuropathy model with Karwinskia humboldtiana (buckthorn) fruit in rats. Histol. Histopathol., Suppl.1:S157-8, 2005.

Salazar-Leal, M.; Flores, M. S.; Sepúlveda-Saavedra, J.; RomeroDíaz, V. J.; Becerra-Verdín, E. M.; Tamez-Rodríguez, V. A.; Martínez, H. R.; Piñeyro-López, A. \& Bermúdez M. V. An experimental model of peripheral neuropathy induced in rats by Karwinskia humboldtiana (buckthorn) fruit. J. Peripher. Nerv. Syst., 11(3):253-61, 2006.

Sepúlveda-Saavedra, J.; Van Der-Klei, I.; Kaiser, I.; Piñeyro-López, A.; Harder, W. \& Veenhuis, M. Studies on the effect of T-514 on the integrity of peroxisomes in methyltrophic yeast. FEMS Microbiol. Lett., 91:207-12, 1992.

Ziegler, D.; Hanefeld, M.; Ruhnau, K. J.; Hasche, H.; Lobisch, M.; Schütte, K.; Kerum, G. \& Malessa, R. Treatment of symptomatic diabetic polyneuropathy with the antioxidant alpha-lipoic acid: a 7-month multicenter randomized controlled trial (ALADIN III Study). ALADIN III Study Group. Alpha-Lipoic Acid in Diabetic Neuropathy. Diabetes Care, 22(8):1296-301, 1999.

\section{Correspondence to:}

Dr. Julio Sepúlveda-Saavedra

Facultad de Medicina de la UANL

Departamento de Histología, AP 1563

Monterrey, N.L. 64400

MÉXICO

Fax 52-82-8348-5477

Email: jusesaa@netscape.net

Received: 15-12-2011

Accepted: 06-03-2012 